\title{
TOWARDS A STANDARD OF MODELLING AnNotations in THE E-Health Domain
}

\author{
Zayneb Mannai ${ }^{1}$, Anis Kalboussi ${ }^{2}$ and Ahmed Hadj Kacem ${ }^{3}$ \\ ${ }^{1}$ ReDCAD Research Laboratory and Faculty of Economics and management, \\ Universityof Sfax, Sfax, Tunisia \\ ${ }^{2}$ ReDCAD Research Laboratory and Higher Institute of computer science and \\ Management, University of Kairouan, Tunisia \\ ${ }^{3}$ Faculty of Economics and management, University of Sfax, Sfax, Tunisia
}

\begin{abstract}
A large number of annotation systems in e-health domain have been implemented in the literature. Several factors distinguish these systems from one another. In fact, each of these systems is based on a separate paradigm, resulting in a disorganized and unstructured vision. As part of our research, we attempted to categorize them based on the functionalities provided by each system, and we also proposed a model of annotations that integrates both the health professional and the patient in the process of annotating the medical file.
\end{abstract}

\section{KEYWORDS}

e-health, annotation system, classification, health record, healthcare professional

\section{INTRODUCTION}

The annotative activity, which involves attaching a note to a text, is frequently employed in a variety of professions, including medicine. Indeed, doctors annotate their patients 'files, which include episodes of disease and treatment paths. Given the importance of medical annotation in patient monitoring and multidisciplinary cooperation, the researchers put out the necessary effort to convert traditional pencil annotation to digital annotation. They subsequently developed annotation systems to help professionals annotate medical record. [1,2]

How many healthcare professionals have struggled to share information with colleagues or transmit it to their patients?

Who among the patients have trouble transmitting their digital medical record data from one doctor to another?

By asking these questions, we can underline the difficulty of interoperability between systems. [3] In fact, there is a communication breakdown among the numerous stakeholders in the digital medical record, limiting both the doctor's and the patient's mobility. Furthermore, each of the medical record annotation systems 'attributes are conceptualized using a specific model, making these systems understandable by information systems. However, the cited models do not match the semantic annotation requirements.

The goal of this study is to provide an organized overview of numerous annotation systems by categorizing them according to their functions and proposing a standard model of annotation that 
incorporates both professionals and patients in medical record annotation.

The following is how the paper is organised: the classification of annotation systems based on their functionalities is highlighted in section 2. The proposed model's conception is presented in section 3. Section 4 closed with a conclusion and some future reflections.

\section{Electronic Medical Record and Medical AnNotation System}

\subsection{Electronic Medical Record}

The computerized medical record is an electronic record that incorporates all textual exchanges between health providers, as well as the synthesis of treatments, diagnoses, and patient followups. In this regard, we can state that the electronic patient file ensures that the treatment protocol is followed, that it contributes to improving the quality of medical treatments, that ensures data security, and that it facilitates coordination amongst interveners by facilitating data access. Furthermore, the patient's access to his medical records enables him to provide clear information regarding his illness and treatment. We can say that the professional's and the patient's interests are intertwined.

\subsection{Annotation Systems}

There are several definitions of annotation in the literature. They vary depending on the research fields:

- [7] defines annotation as "the process of making remarks on a text in order to explain or comment on it".

- In the context of Human-Machine interactions research, the annotation is defined as a comment concerning a perceived object that is distinguishable both for the commentator and for the reader who interprets it. [8]

- In semantic web research, the $\mathrm{W} 3 \mathrm{C}$ indicates that annotation can take the form of metadata because it generates data in addition to other data. [9]

- The annotation is the result of an active reading of the data on the web. In fact, it is usedto identify the points that appear important to the reader in order to aid his comprehension of the text. [10]

- An annotation is used in the field of medical biology to define the functions and different coding regions of the genome. It is utilized to explain the significance of the genomes.[11]

- The medical annotation is a type of annotation used by healthcare professionals of various specialities to mark data pertaining to a specific patient. [1]

\subsection{Type of Medical Annotation Object}

Annotation can be divided into two categories: [12]

- Cognitive annotation: this annotation has an observable form on the document. It is used by human agents and thus necessitates a cognitive and mental effort to comprehend.

- Computational annotation: also known as 'meta-data'. Software agents are in charge of treating and manipulating it. The metadata allows us to annotate. Computer resources to make them easier for the machine to utilise. 


\subsection{Categories of Computer Annotation Activitie}

To begin the annotative activity, select the anchor and annotation shape from the annotation software toolbar. The annotation must finally meet all of the required properties by being fixed to a well-defined target. This annotation procedure consists of three forms: [12]

- Manual form: the entire annotation process is solely the user's responsibility. He selects the shape of the annotation, then the anchor and finally the annotation. This type of annotative activity is comparable to that done on paper.

- Automatic form: the machine is programmed to carry out the entire annotation process without the need for human intervention. The annotations are aided by the use of context sensors and pattern recognition techniques.

- Semi-automatic form: the user initiates the annotating process. Once the user has chosen an annotation mode, the system automatically generates annotations based on a model developed with rules in development. At this level, the user merely enters to confirm and refine the rules. No human assistance is required after a certain stage.

\subsection{Annotation Systems Status}

- Plug-in: is small complementary program that adds new features. It is used in many programs and apps.

- Web-site: is a collection of web pages and resources. Some websites are designed to annotate resources that users consult.

- Application: it is program or set of software used to complete a task. The annotation can be carried out by an application which offers certain functionalities.

\subsection{Annotation Systems Functionalities}

Annotation systems come with a variety of features. We divide them into three groups: memorization features, reuse features and sharing. [1,13]

- $\quad$ Sharing features:

F1: Annotation export: the annotator wishes to send all or a portion of the annotations that have been written on a document.

F2: Annotation import: the user can receive annotations. This feature enables him to add new annotations to a document as if it had been annotated by two different annotators.

- Memorization features:

F3: Reading and browsing the document: access to the document should be granted to the user. If that's the case, the reader opens the annotation system and chooses an existing document. He can use the mouse, keyboard arrows, and the elevator to navigate to the next and previous pages, as well as the beginning on finish of each page.

F4: Annotation creation: there are two methods for creating annotations:

- Tool/object mode: the annotator chooses a type of annotation and applies it to the content.

- Object/tool mode: the annotator chooses a target and then executes an annotation command. 
F5: Annotation modification: the annotator has the ability to change all of the annotation's parameters (shape, colour, content etc.).

F6: Delete of annotation: the annotation can be removed without being archived.

F7: Visualization of the annotation in the document: the annotations are scatteredthroughout the main document.

\section{- $\quad$ Reuse features:}

F8: Filtering: the reader is looking for one or more annotations that meet certain requirements.

F9: Visualization of the annotation outside the document: annotations are displayed in adifferent location than the primary document.

F10: Sorting definition: the reader organizes the list of displayed annotations by sorting them based on their attributes.

F11: Merging of annotated documents: This tool lets the user build a report with annotated documents. Based on the annotation, the merging produces a summary of the patient's condition. This process enables experts to share documents.

F12: Comparison of annotations: this comparison seeks to determine whether or not twogiven annotations have the same meaning.

F13: Redefinition of an annotation: the practitioner manually traces any annotation, and then the machine automatically intervenes to retrace it.

F14: Annotation extraction: an annotation can be saved in a variety of formats (text, XML, etc.).

F15: Linking the annotation to an external source: annotated content is a link to an external source, and the annotation is a link to that source.

F16: Localization of the annotation and calculation of the area of the annotated zone: this functionality allows the user to specify the coordinates of the anomalous component(sick) and determine its interface by locating the annotation and calculating the area of the annotated zone.

Table 1. Features of annotation systems

\begin{tabular}{|c|c|c|c|c|c|c|c|c|c|c|c|c|c|c|c|c|}
\hline $\begin{array}{c}\text { Name of } \\
\text { annotation } \\
\text { system }\end{array}$ & F1 & F2 & F3 & F4 & F5 & F6 & F7 & F8 & F9 & F10 & F11 & F12 & F13 & F14 & F15 & F16 \\
\hline $\begin{array}{c}\text { Automatic } \\
\text { annotator [14] }\end{array}$ & & $*$ & $*$ & $*$ & $*$ & $*$ & $*$ & $*$ & $*$ & $*$ & & & & $*$ & \\
\hline Octane [15] & & $*$ & $*$ & $*$ & $*$ & $*$ & $*$ & $*$ & $*$ & $*$ & & & & \\
\hline CUPP [16] & & $*$ & $*$ & $*$ & $*$ & $*$ & $*$ & $*$ & $*$ & $*$ & & & & \\
\hline SS [17] & & $*$ & $*$ & $*$ & $*$ & $*$ & $*$ & $*$ & $*$ & & & & & \\
\hline $\begin{array}{c}\text { RIL- } \\
\text { CONTOUR[18] }\end{array}$ & & $*$ & $*$ & $*$ & $*$ & $*$ & $*$ & & & & & & & \\
\hline RITAN [19] & & $*$ & $*$ & $*$ & $*$ & $*$ & $*$ & & & & & & & \\
\hline RNAmod \\
{$[20]$}
\end{tabular}


Health Informatics - An International Journal (HIIJ) Vol.10, No.4, November 2021

\begin{tabular}{|c|c|c|c|c|c|c|c|c|c|c|c|c|c|c|c|c|}
\hline Crowd flower[23] & & & $*$ & $*$ & * & $*$ & $*$ & & $*$ & $*$ & & & & & & \\
\hline IOG Gran [24] & & & $*$ & $*$ & * & $*$ & $*$ & & $*$ & & & & & & & $*$ \\
\hline Sonto [25] & 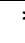 & $*$ & $*$ & $*$ & $*$ & $*$ & $*$ & & $*$ & & & & & & & \\
\hline $\begin{array}{l}\text { RIL- } \\
\text { CONTOUR[26] }\end{array}$ & & & $*$ & $*$ & $*$ & $*$ & $*$ & $*$ & $*$ & & & & & & $*$ & $*$ \\
\hline ROMEDI [27] & & & $*$ & $*$ & $*$ & $*$ & $*$ & & $*$ & & & & & & & \\
\hline $\mathrm{QA}[28]$ & & & $*$ & $*$ & * & $*$ & $*$ & $*$ & $*$ & & & $*$ & & & & \\
\hline CB13 [29] & & & $*$ & $*$ & $*$ & $*$ & $*$ & & $*$ & & & & & & & \\
\hline Med3D [30] & & & $*$ & $*$ & $*$ & $*$ & $*$ & & $*$ & & & & $*$ & & * & * \\
\hline $\begin{array}{l}\text { Image annotator } \\
{[31]}\end{array}$ & & & $*$ & $*$ & $*$ & $*$ & $*$ & & $*$ & & & & & & $*$ & $*$ \\
\hline $\begin{array}{l}\text { 3DBIONOTE } \\
\text { S[32] }\end{array}$ & & $*$ & $*$ & $*$ & $*$ & $*$ & $*$ & $*$ & $*$ & & & & & & & \\
\hline LERUDI [33] & & & $*$ & $*$ & * & $*$ & $*$ & $*$ & $*$ & & & & & & & \\
\hline Micro MD [34] & & & $*$ & $*$ & * & & $*$ & & $*$ & & & & & & & \\
\hline BestSlice [35] & 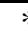 & $*$ & $*$ & $*$ & $*$ & $*$ & $*$ & $*$ & $*$ & & & & & & & \\
\hline Verdant [36] & & & $*$ & $*$ & $*$ & $*$ & $*$ & & $*$ & & & & & & & \\
\hline $\begin{array}{c}\text { 2017: } \\
\text { PRETEXT[37] }\end{array}$ & & & $*$ & $*$ & $*$ & $*$ & $*$ & & $*$ & & & & & & & \\
\hline $\begin{array}{c}\text { SIFR BIOPORTA } \\
\text { L[38] }\end{array}$ & & & $*$ & $*$ & $*$ & $*$ & $*$ & & $*$ & & & & & & & \\
\hline $\begin{array}{l}\text { ODMSummary } \\
{[39]}\end{array}$ & & & $*$ & $*$ & $*$ & * & $*$ & & $*$ & & & * & & & & \\
\hline GIDAC [40] & & & * & $*$ & * & $*$ & $*$ & & $*$ & & & & & & $*$ & $*$ \\
\hline VCFminer [41] & & & $*$ & $*$ & $*$ & $*$ & $*$ & & $*$ & & $*$ & & & & & \\
\hline$\overline{\text { CART [42] }}$ & & & $*$ & $*$ & * & $*$ & $*$ & & $*$ & & & & & & & \\
\hline Lead tools[43] & & & $*$ & $*$ & * & $*$ & $*$ & & $*$ & & & & & & & $*$ \\
\hline
\end{tabular}

\section{Proposed Model}

Our contribution consists of giving a formal model of annotation to aid in the solution of the interoperability challenge. Various annotation record models have been proposed in the literature. We will use as examples: HL7 (Health Level Seven) [44], CDA (Clinical Document Architecture) [45], XDS (Cross Enterprise Document Sharing) [46], DICOM (Digital Image 
Communication in Medicine) [47] and SNOMED (Systematized Nomenclature of medicine) [48].In this regard, we can also quote the work of [49], who suggested an annotation model that considers the semantics of the annotation but overlooks some details. Only healthcare professionals are included in the annotation of medical files in this model, whereas, as previously stated, the patient has the right to access his file, as well as documents relating to the diagnoses and treatments that concern him, and can also make annotations in his own personal space of expression.

The strategy of annotation may change based on the healthcare professional's speciality as well as the patient's degree of learning. By using this strategy, the patient must be able to comprehend his treatment regimen at all phases of the care cycle, including the required prescriptions and the doctor's recommendations. To carry out our quest and achieve our targeted goals of ensuring systems interoperability, we obviously select the advantages of the models mentioned and attemptto overcome their limitations. By including the patient in the annotation of his or her own record, building a healthcare cycle, and encouraging annotation semantics, our research proposes a novel architecture for the many components of annotation in the electronic medical record.

The proposed model (figure 1) contains the following concepts:

- Visual aspect: represents how the annotation appears in the document.

- Anchor: represents the annotation's location in the document.

- Content: the annotator's mental representation as it has evolved.

- Target: it is the foundation of the annotation that can be (a set of documents, a single document or a part of document).

- Environmental aspect: encompasses all aspects of interaction with environment.

- Spatial-temporal framework: designates the date and location of the act of annotation.

- Tool: the hardware instrument that was utilized to make the annotation.

- Validity: specifies the date on which a task begins, ends or all cycle date.

- Semantic aspect: refers to the features that allow the annotation to be tailored to Its intended use.

- Annotation theme: designates the annotation's communication object.

- Annotation objective: reveals the annotator's aims through his annotation act.

- Reading objective: indicates the reader's expectations based on his reading and comprehension of the information.

- Reading domain: this is the domain in which the user reads the content and annotates it.

- Stakeholders: anyone who has the patient's permission to see and annotate the patient's file.

- Collection of documents: the target of the annotation can be a collection of documents, a document or a section of a document.

- Healthcare professional: An individual who is qualified and permitted to give healthcare services to patients and who is associated with a particular specialty or profession. Aside from that, he is a stakeholder with the capability of annotating a patient's health record..

- Patient: The patient has the right to access and annotate his medical file based on his level of learning, specific needs, and, ultimately, his profile. 
Health Informatics - An International Journal (HIIJ) Vol.10, No.4, November 2021

- Treatment course: the purpose of the integrated care path is to give each patient with individualized medical monitoring, medical file management, and prevention. There are three stages to the therapeutic experience (discovery, examination, follow-up and control).

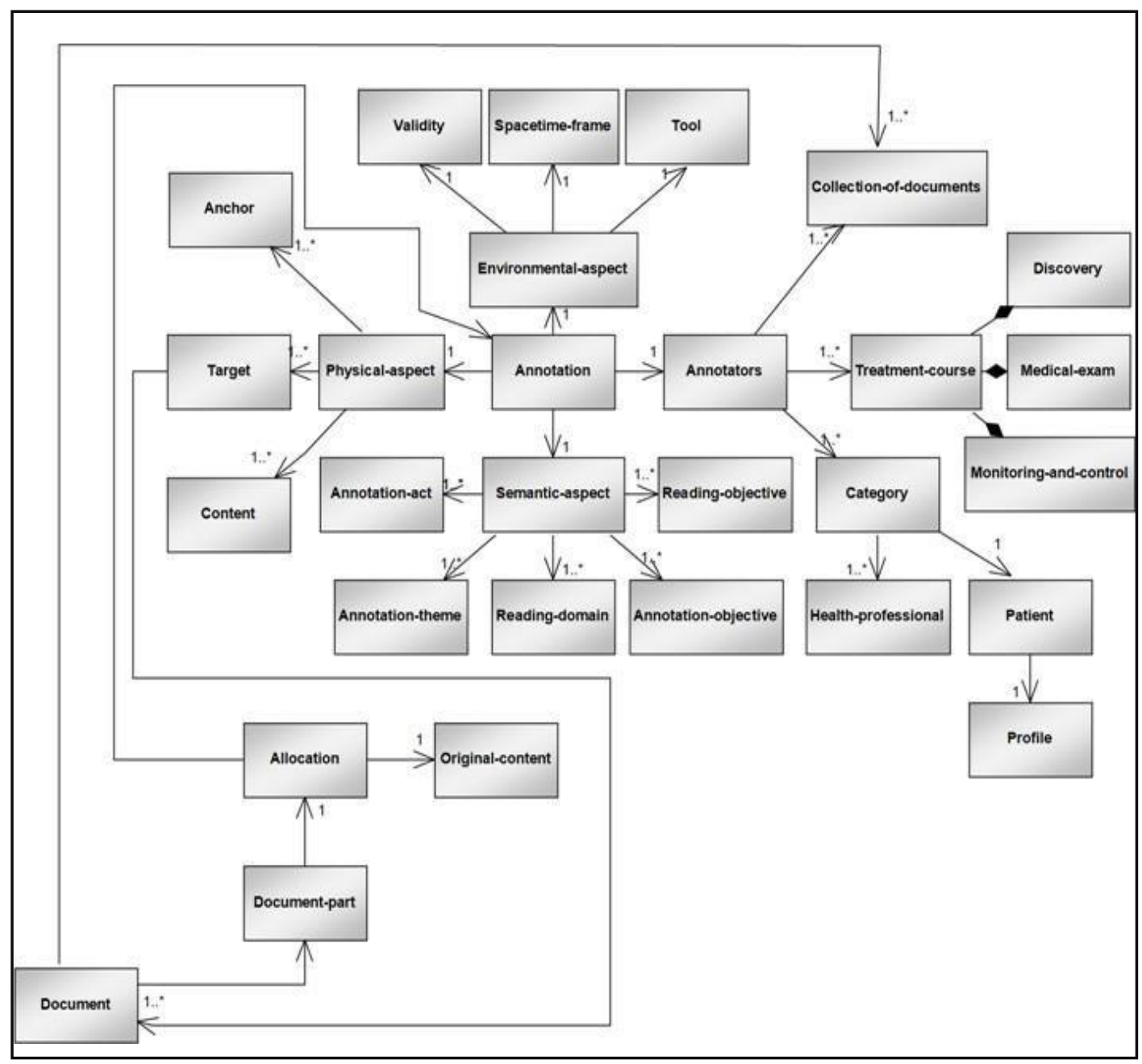

Figure 1. proposed annotation model

\section{CONCLuSions}

In conclusion, it is clear that this research has demonstrated a consistent and coherent classification of digital health annotation systems. This classification, which is based on the features supplied by each annotation system, makes it easier to identify constraints and potential issues in the medical annotation systems field. In light on this, we developed a new ontological model that engages the patient in the annotation of his medical records which helps to develop a more sophisticated annotation system. Our research will now go on to developing an annotation system based on the proposed model. 
Health Informatics - An International Journal (HIIJ) Vol.10, No.4, November 2021

\section{ACKNOWLEDGEMENTS}

The authors would like to thank all members of ReDCAD Research Laboratory, Sfax, Tunisia.

\section{REFERENCES}

[1] Chehab, K., Kalboussi, A., \& Kacem, A. H. (2018, July). Study of Annotations in e-health Domain. InInternational Conference on Smart Homes and Health Telematics (pp. 189-199).Springer, Cham.

[2] Bringay, S. (2006). Les annotations pour supporter la collaboration dans le dossier patientélectronique (Doctoral dissertation, Université de Picardie Jules Verne).

[3] Kalboussi, A., Mazhoud, O., \& Kacem, A. H. (2016). Comparative study of web annotation systems used by learners to enhance educational practices: features and services. International Journal of Technology Enhanced Learning, 8(2), 129-150.

[4] Kalboussi, A., Mazhoud, O., Hadj Kacem, A., \& Omheni, N. (2013, November). A formal model of learner's annotations dedicated to web services invocation. In Proceedings of the 21st International Conference on Computers in Education (ICCE 2013) (pp. 166-169).

[5] Chehab, K., Kalboussi, A., \& Kacem, A. H. (2020, June). Study of Healthcare Professionals' Interaction in the Patient Records Based on Annotations. In International Conference on SmartHomes and Health Telematics (pp. 316-328). Springer, Cham.

[6] Jedwab, R. M., Chalmers, C., Dobroff, N., \& Redley, B. (2019). Measuring nursing benefits of an electronic medical record system: A scoping review. Collegian, 26(5), 562-582.

[7] Desmontils, E., \& Jaquin, C. (2002). Indexing a web site with a terminology-oriented ontology, The Emerging Semantic Web, IF Cruz S. Decker J. Euzenat and DL McGuinness Ed.

[8] Baldonado, M., Cousins, S., Gwizdka, J., \& Paepcke, A. (2000, September). Notable: At the intersection of annotations and handheld technology. In International Symposium on Handheld and Ubiquitous Computing (pp. 100-113). Springer, Berlin, Heidelberg.

[9] Sanderson, R., Ciccarese, P., \& Van de Sompel, H. (2013, May). Designing the W3C open annotation data model. In Proceedings of the 5th Annual ACM Web Science Conference (pp.366-375).

[10] Prié, Y., \& Garlatti, S. (2004). Méta-données et annotations dans le Web sémantique. Revue I3 Information-Interaction-Intelligence, 4, 45-68.

[11] Stein, L. (2001). Genome annotation: from sequence to biology. Nature reviews genetics, 2(7), 493503.

[12] Kalboussi, A., Omheni, N., Mazhoud, O., \& Kacem, A. H. (2015, September). How to organize the annotation systems in human-computer environment: study, classification and observations. In IFIP Conference on Human-Computer Interaction (pp. 115-133). Springer, Cham.

[13] Mille, D. (2005). Modèles et outils logiciels pour l'annotation sémantiquede documentspédagogiques (Doctoral dissertation, Université Joseph-Fourier-Grenoble I).

[14] Krsnik, I., Glavaš, G., Krsnik, M., Miletić, D., \& Štajduhar, I. (2020). Automatic Annotation of Narrative Radiology Reports. Diagnostics, 10(4), 196.

[15] Zeng, J., Shufean, M. A., Khotskaya, Y., Yang, D., Kahle, M., Johnson, A., ... \& Meric- Bernstam, F. (2019). OCTANE: oncology clinical trial annotation engine. JCO clinical cancerinformatics, 3, 1-11.

[16] Barrett, K., \& Lange, L. (2019). Peptide-based functional annotation of carbohydrate-active enzymes by conserved unique peptide patterns (CUPP). Biotechnology for biofuels, 12(1), 1-21.

[17] Hong, J., Luo, Y., Mou, M., Fu, J., Zhang, Y., Xue, W., ... \& Zhu, F. (2020). Convolutional neural network-based annotation of bacterial type IV secretion system effectors with enhanced accuracy and reduced false discovery. Briefings in bioinformatics, 21(5), 1825-1836.

[18] Philbrick, K. A., Weston, A. D., Akkus, Z., Kline, T. L., Korfiatis, P., Sakinis, T., ... \& Erickson,

B. J. (2019). RIL-contour: a medical imaging dataset annotation tool for and with deeplearning. Journal of digital imaging, 32(4), 571-581.

[19] Zimmermann, M. T., Kabat, B., Grill, D. E., Kennedy, R. B., \& Poland, G. A. (2019). RITAN:rapidintegration of term annotation and network resources. PeerJ, 7, e6994.

[20] Liu, Q., \& Gregory, R. I. (2019). RNAmod: an integrated system for the annotation of mRNAmodifications. Nucleic acids research, 47(W1), W548-W555.

[21] (2018). Retrieved from http://www.medicalip.com/en

[22] Ma, J., \& Meng, J. (2018, June). Interactive genomic visualization for R/bioconductor. In BIBE 2018; International Conference on Biological Information and Biomedical Engineering (pp. 14).VDE. 
Health Informatics - An International Journal (HIIJ) Vol.10, No.4, November 2021

[23] (2018). Retrieved from https://www.ncbi.nlm.nih.gov/pmc/articles/PMC4299942

[24] (2018). Retrieved from http://iogram.ca

[25] (2018). Retrieved from http://aclweb.org/anthology/P18-4012

[26] Philbrick, K. A., Weston, A. D., Akkus, Z., Kline, T. L., Korfiatis, P., Sakinis, T., ... \& Erickson,

B. J. (2019). RIL-contour: a medical imaging dataset annotation tool for and with deeplearning. Journal of digital imaging, 32(4), 571-581.

[27] Cossin, S., Loustau, R., Jouhet, V., Létinier, L., Mougin, F., Evrard, G., ... \& Thiessard, F. (2018). ROMEDI, une terminologie médicale française pour la détection des médicaments en texte libre. Artificial Intelligence Platform, Nancy.

[28] Kilicoglu, H., Abacha, A. B., Mrabet, Y., Shooshan, S. E., Rodriguez, L., Masterton, K., \& DemnerFushman, D. (2018). Semantic annotation of consumer health questions. BMC bioinformatics, 19(1), $1-28$.

[29] Berrios, L., \& Ely, B. (2018). Achieving accurate sequence and annotation data for Caulobacter vibrioides CB13. Current microbiology, 75(12), 1642-1648.

[30] Lavrič, P., Bohak, C., \& Marolt, M. (2017, May). Collaborative view-aligned annotations in webbased 3D medical data visualization. In 2017 40th International Convention on Information and Communication Technology, Electronics and Microelectronics (MIPRO) (pp. 259-263). IEEE.

[31] Gordo, A., Almazan, J., Revaud, J., \& Larlus, D. (2017). End-to-end learning of deep visual representations for image retrieval. International Journal of Computer Vision, 124(2), 237-254.

[32] Segura (2017). Bioinformatique, $33: 3655-3657$

[33] Gayet, P., Charlet, J., Janin, N., Clout, E., Decleck, G., \& Miroux, P. (2017). Une synthèse du dossier médical pour décider aux urgences: le projet LERUDI. Annales françaises de médecine d'urgence, 7(3), 166-173.

[34] Lavrič, P., Bohak, C., \& Marolt, M. (2017, May). Collaborative view-aligned annotations in webbased 3D medical data visualization. In 2017 40th International Convention on Information and Communication Technology, Electronics and Microelectronics (MIPRO) (pp. 259-263). IEEE.

[35] (2017). Retrieved from https://www.best.edu.au/s/5njv62ar?data=8\%400!9\%4028320!10\%40$27251.5 \&$ version $=1$

[36] McKain, M. R., Hartsock, R. H., Wohl, M. M., \& Kellogg, E. A. (2017). Verdant: automated annotation, alignment and phylogenetic analysis of whole chloroplast genomes. Bioinformatics, 33(1), 130-132.

[37] Towbin, A. J., Meyers, R. L., Woodley, H., Miyazaki, O., Weldon, C. B., Morland, B., ... \& Tiao, $\mathrm{G}$.

M. (2018). 2017 PRETEXT: radiologic staging system for primary hepatic malignancies ofchildhood revised for the Paediatric Hepatic International Tumour Trial (PHITT). Pediatric radiology, 48(4), 536-554.

[38] Jonquet, C., Annane, A., Bouarech, K., Emonet, V., \& Melzi, S. (2016, June). SIFR BioPortal: Un portail ouvert et générique d'ontologies et de terminologies biomédicales françaises au service de l'annotation sémantique. In JFIM: Journées Francophones d'Informatique Médicale.

[39] Storck, M., Krumm, R., \& Dugas, M. (2016). ODMSummary: a tool for automatic structured comparison of multiple medical forms based on semantic annotation with the Unified Medical Language System. PloS one, 11(10), e0164569.

[40] Vizza, P., Guzzi, P. H., Veltri, P., Cascini, G. L., Curia, R., \& Sisca, L. (2016, December). GIDAC: a prototype for bioimages annotation and clinical data integration. In 2016 IEEE International Conference on Bioinformatics and Biomedicine (BIBM) (pp. 1028-1031). ieee.

[41] Hart, S. N., Duffy, P., Quest, D. J., Hossain, A., Meiners, M. A., \& Kocher, J. P. (2016). VCF- Miner: GUI-based application for mining variants and annotations stored in VCF files. Briefings in bioinformatics, 17(2), 346-351.

[42] Deghou, S., Zeller, G., Iskar, M., Driessen, M., Castillo, M., van Noort, V., \& Bork, P. (2016). CART - a chemical annotation retrieval toolkit. Bioinformatics, 32(18), 2869-2871.

[43] (2016). Retrieved from https://www.leadtools.com/sdk/annotation

[44] Quinn, J. (1999). An HL7 (health level seven) overview. Journal of AHIMA, 70(7), 32-4.

[45] Müller, M. L., Ückert, F., Bürkle, T., \& Prokosch, H. U. (2005). Cross-institutional data exchange using the clinical document architecture (CDA). International journal of medical informatics, 74(2-4), 245-256.

[46] Wozak, F., Ammenwerth, E., Breu, M., Penz, R., Schabetsberger, T., Vogl, R., \& Wurz, M. (2006). Medical Data GRIDs as approach towards secure cross enterprise document sharing (based on IHE 
Health Informatics - An International Journal (HIIJ) Vol.10, No.4, November 2021

XDS). Studies in health technology and informatics, 124, 377-383.

[47] Pianykh, O. S. (2012). What is DICOM?. In Digital Imaging and Communications in Medicine (DICOM) (pp. 3-5). Springer, Berlin, Heidelberg.

[48] Rothwell, D. J. (1980). SNOMED (Systematized Nomenclature of Medicine). Microglossary for surgical pathology. Skokie, IL: College of American Pathologists.

[49] Chehab, K., Kalboussi, A., \& Kacem, A. H. (2020, June). Study of Healthcare Professionals' Interaction in the Patient Records Based on Annotations. In International Conference on SmartHomes and Health Telematics (pp. 316-328). Springer, Cham.

\section{AUTHORS}

Zayneb Mannai: Phd student in Faculty of Economics and management, University of Sfax, Sfax, Tunisia.

Anis Kalboussi: Associate professor in Higher Institute of computer science and Management, University of Kairouan, Tunisia.

Ahmed Hadj Kacem: Professor in Faculty of Economics and management, University of Sfax, Sfax, Tunisia. 